\title{
Waste to watt: Unwanted solid materials formation, transport and cycling in biogas engine systems
}

\author{
AHMAD AMIR SHABRIN BIN MOHD KHAIDI ${ }^{1}$, \\ ERZSÉBET HARMAN-TÓTH ${ }^{1}$, IMRE GÖNCZI ${ }^{2}$, TAMÁS \\ MIREISZ $^{3}$, TAMÁS KÖVÁRI ${ }^{3}$ AND TAMÁS GÁBOR \\ WEISZBURG $^{1}$
}

${ }^{1}$ Eötvös Loránd University

${ }^{2} \mathrm{NRG}$ Group

${ }^{3}$ Budapest Waterworks

Presenting Author: ameershabreen@gmail.com

Green energy production from biogas is more and more preferred to depleting fossil fuel resources. Biogas engines utilize biogas produced from two main sources, urban waste landfill and wastewater treatment plant to produce heat and electricity at the same time. Other than biogas as fuel, vital components of the engine systems include the air (to be mixed with biogas) for the combustion and lubricating oil for the machinery parts.

The flaw in biogas comes from its inherent compositional variability. Detrimental $\mathrm{H}_{2} \mathrm{~S}$ and siloxanes, trace components inside biogas, decompose during the combustion of $\mathrm{CH}_{4}$, leading to the deposition of solid crust in the combustion chamber. Characterization of these materials revealed the presence of both glassy (Ca-Si-O-S composition) and crystalline phases $\left(\mathrm{SiO}_{2}\right.$ polymorphs, anhydrite). Formed under high pressure and temperature, the accumulating material will gradually melt in the more and more overheated chamber and finally solidify as combustion stops for maintenance.

Lubricating oil plays an important role in maintaining the engine, it is the first indicator (routine test) to assess engine conditions. It is a connecting medium between all the machinery parts in the engines that may sample the deposits of the combustion chamber. Currently, we trace the presence of deposit particles inside the lubricating oil. We used debris filter method for the extraction of solids from the used oil samples. The size of the particles inside the oil cannot be more than $30 \mu \mathrm{m}$ in normal working conditions.

SEM revealed the presence of various particles of $\mathrm{Si}, \mathrm{Ca}, \mathrm{S}$, $\mathrm{Na}, \mathrm{K}, \mathrm{P}$ elemental composition in the 20-30 um size range, verifying that also the solids formed inside the combustion chamber are transferred to the lubricating oil. These problems lead to the increased oil consumption of the systems and decrease its lifespan.

To develop a strategical approach in green technology development, the problems faced by biogas-to-energy industry need to be studied as a whole from biogas input till the end line with the exhaust production.

This work was completed in the ELTE Institutional Excellence Program (TKP2020-IKA-05), financed by the Hungarian Ministry of Human Capacities, and was supported by the Koch Sándor Foundation. 